\title{
Rate of energy absorption by a closed ballistic ring
}

\author{
Doron Cohen ${ }^{1}$, Tsampikos Kottos ${ }^{2,3}$ and Holger Schanz ${ }^{3,4}$ \\ ${ }^{1}$ Department of Physics, Ben-Gurion University, Beer-Sheva 84105, Israel \\ ${ }^{2}$ Department of Physics, Wesleyan University, Middletown, Connecticut 06459-0155, USA \\ ${ }^{3}$ Max-Planck-Institut för Dynamik und Selbstorganisation Göttingen, Germany \\ ${ }^{4}$ Institut für Nichtlineare Dynamik, Universität Göttingen, Germany
}

\begin{abstract}
We make a distinction between the spectroscopic and the mesoscopic conductance of a closed ring. We show that the latter is not simply related to the Landauer conductance of the corresponding open system. A new ingredient in the theory is related to the non-universal structure of the perturbation matrix which is generic for quantum chaotic systems. These structures may created bottlenecks that suppress the diffusion in energy space, and hence the rate of energy absorption. The resulting effect is not merely quantitative: For a ring-dot system we find that a smaller Landauer conductance implies a smaller spectroscopic conductance, while the mesoscopic conductance increases. Our considerations open the way towards a realistic theory of dissipation in closed mesoscopic ballistic devices.
\end{abstract}

\section{Introduction}

When a physical system is subjected to an external perturbation it can absorb energy from the driving source (Fig. 1). The rate of this absorption depends crucially on the internal dynamics. Here we are concerned with a mesoscopic electronic system. Mesoscopic means that the electrons must be treated as quantum mechanical particles whose wavelength is small compared to the classical dimensions. In such circumstances, which assume long coherence time, it is important whether or not the resulting electron dynamics would be integrable or chaotic or diffusive if approximated classically, and the shape of the device becomes relevant. The fingerprints of such nonuniversal (semiclassical) effects have been found in numerous experiments with open mesoscopic systems. It is our objective to extend this idea into the realm of closed mesoscopic systems, in the context of (semi-linear) response theorył.

Main observation: In this paper we expose a new ingredient in the theory of energy absorption by closed mesoscopic driven systems. The main idea is that there are circumstance in which the rate of absorption depends on the possibility to make long sequences of transitions. The possibility to make a connected sequence of transitions between energy levels is greatly affected by structures in the energy landscape of the device. These structures are the fingerprint of the "shape" of the device, and more generally they are implied by semiclassical considerations. In the quantum chaos literature such structures are termed non-universal so as to distinguish them

$\ddagger$ The response of chaotic systems to weak driving is "linear" in the classical treatment. We look for a quantum mechanical related departure from linear response theory. This should be contrasted with the traditional studies of "quantum chaos" models (such as the 'quantum kicked rotator') where in the absence of driving the system is integrable(!) and consequently the response is manifestly non-linear both classically and quantum mechanically. 
from the universal fluctuations which are described by random matrix theory. In the context of energy absorption the most important non-universal effect is the presence of "bottlenecks" in energy space where the couplings between levels are small. The rate of energy absorption can be greatly reduced by the presence of such bottlenecks. In order to take the effect of these structures into account we have to go beyond the conventional framework of linear response theory (LRT).

Main application: Closed mesoscopic rings are of great interest [1, 2, 3, 4, 5, 6. 7, 8, 9]. For such devices the relation between the conductance and the internal dynamics is understood much less [10] than for open systems. This is not surprising if one realizes that the theoretical analysis of $G$ relies on completely different concepts in the open and in the closed case. For an open systems the Landauer formula expresses the conductance $G_{L}$ in terms of the scattering matrix of the device. This is a very convenient starting point for a subsequent analysis since it is not necessary to account for the dissipation of energy explicitly. By contrast, for closed systems the mechanism for dissipation is an issue. Conventionally one assumes a weak coupling to an external bath which leads to a steady state but preserves approximately the main features of the internal dynamics.

First measurements of the conductance of closed mesoscopic rings have been reported more than a decade ago [9]. In a typical experiment a collection of mesoscopic rings are driven by a time dependent magnetic flux $\Phi(t)$ which creates an electromotive-force $(\mathrm{EMF})-\dot{\Phi}$ in each ring. Assuming that Ohm's law applies, the induced current is $I=-G \dot{\Phi}$ and consequently the rate of energy absorption is given by Joule's law as

$$
\dot{\mathcal{W}} \equiv \text { Rate of energy absorption }=G \dot{\Phi}^{2}
$$

where $G$ is called the conductance§. One should be very careful with the terminology here. We neither consider "two terminal measurement" of the conductance nor "four terminal measurement". One may say that closed ring is a "zero terminal" device (no leads). So when we say "conductance" we relate to the coefficient $G$ in Eq.(I). In practice $G$ is deduced form a measurement of the magnetic susceptibility $\chi(\omega)$. If we have a large collections of rings then $G$ should be identified as the $\omega \rightarrow 0$ limit of $\operatorname{Im}[\chi(\omega)] / \omega$ divided by the number of rings. We are aware that some of the people in the condensed matter community avoid the use of the term "conductance" for a zero terminal device. There are no leads attached, so a transport measurement in the sense of the Landauer geometry is not applicable. However, we are not aware of a better name for $G$ of Eq.(11). We think that the terminology issue is mainly a matter of taste. Namely, if the ring were 1 meter in diameter, rotating between the magnets of a commercial generator, no one would be bothered by calling $G$ "conductance", so why not to adopt the same terminology in the nano scale?

In typical circumstances, which we define more precisely later on, the energy absorption process is dominated by Fermi-Golden-Rule (FGR) transitions, and the strong dynamical localization effect [5] is irrelevant. We shall always assume this FGR regime and neglect other dissipation mechanisms such as Landau-Zener transitions [1] or Debye relaxation 2]. The FGR picture with some extra assumptions (that we would like to challenge) is the basis for LRT. It leads to the Kubo formula, which is a major tool in many fields of theoretical physics and Chemistry. Diagrammatic

$\S$ The terminology of this paper, and in particular our notion of "conductance" are the same as in the theoretical review [8] and in the experimental work [9]. 
techniques of calculating conductance are based on this formula.

Past works: In the case of diffusive rings the Kubo formula leads to the Drude formula for $G$. A major challenge for past studies was to calculate and to measure weak localization corrections to the Drude result, taking into account the level statistics and the type of occupation. For a review see [8]. It should be clear that these corrections do not change the leading order Kubo-Drude result.

Present work: In the case of ballistic rings we would like to argue that even if the FGR picture of energy absorption applies, still there are circumstance where the Kubo formula, and hence also the Drude result, fail even as a rough approximation. It should be clear that our theory is not in contradiction with LRT. Rather it goes beyond LRT, and reduces to LRT in the appropriate limit. The failure of the Kubo formula is related to non-universal features of the energy landscape which are implied by having a mean free path larger than the size of the system. To some extent our theory is inspired by ideas from percolation theory: but the percolation is in energy space rather than in real space.

Scope: In this paper we analyze an example of a single mode device, while in a follow-up work 12] we analyze an example of a multi-mode ring. The single mode example of this paper (unlike the multi mode example of [12]) possibly seems somewhat artificial. Its advantage is its simplicity. Our interest in this paper is to clarify the main idea of the conductance calculation with one simple prototype example, rather than exploring the full range of possibilities. By now another non-trivial extension of the theory has been worked out in Ref. 13], where the absorption of small metallic grains is calculated. The latter reference has suggested to describe the outcome of the theory as "semilinear response".

Outline of this paper: This paper consists of two parts. In the first part (sections 2-9) we present the general theoretical consideration. In particular:

- We define the notion of "conductance" in the context of closed systems. This leads to our distinction between mesoscopic and spectroscopic conductance.

- We state our main results regarding the conductance of a single mode ballistic device, and discuss their experimental significance.

- We review the FGR picture, and explain the emergence of diffusion in energy space, and the associated dissipation effect.

- We clarify the main ingredient in our theory, which is the calculation of the coarse grained diffusion

- We derive a formula for the mesoscopic conductance assuming a modulated energy landscape.

In the second part we focus on a specific example: a single mode ring-dot model. We express the spectroscopic and the mesoscopic conductance as a function of the averaged Landauer conductance of the corresponding open system. We explore the dependence of the result on the level broadening $\Gamma$. Finally we summarize some key observations and point out again the limitations on the validity of our results. 


\section{Mesoscopic versus Spectroscopic conductance}

For the understanding of this paper it is crucial to make a distinction between the spectroscopic conductance $G_{\text {spec }}$ and the mesoscopic conductance $G_{\text {meso }}$. Both are defined as the dissipation coefficient which appears in Eq.(11), but they relate to different circumstances. The bottom line is that $G_{\text {spec }}$ is the measured value of $G$ for small EMF, while $G_{\text {meso }}$ is the measured value of $G$ for large EMF. In later sections we shall explain that $G_{\text {spec }}$ can be calculated using the traditional version of the Kubo formula, while $G_{\text {meso }}$ requires a different recipe. Thus it should be clear that our theory does not contradict LRT but rather covers a regime where LRT is no longer valid.

In order to explain the physical picture of energy absorption we refer to the block diagram of Fig. 1. On the one hand the driving source induce transitions between energy levels of the system, leading to an absorption of energy with some rate $\dot{\mathcal{W}}$. On the other hand the system can release energy to some bath (phonons or the surrounding), which leads to a "heat flow" with some rate $\dot{\mathcal{Q}}$. As the system heats up a steady state is reached once $\dot{\mathcal{Q}}=\dot{\mathcal{W}}$.

It is essential to realize that the levels of the system are effectively "broadened" due to the non-adiabaticity of the driving [14] or due to the interaction with the noisy environment [3]. Later we quantify this effect by introducing the level broadening parameter $\Gamma$. One should not make a confusion between

$$
\begin{aligned}
\Gamma & =\text { level broadening parameter in the theory } \\
\gamma_{\mathrm{rlx}} & =\text { relaxation rate towards equilibrium }
\end{aligned}
$$

The above distinction is somewhat analogous to the notions of $1 / T_{2}$ and $1 / T_{1}$ in NMR studies. The parameter $\Gamma$ is essential in order to analyze the induced FGR transitions between levels. Therefore it will appear explicitly in the theoretical derivations. We are going to argue that the rate of energy absorption $\dot{\mathcal{W}}$ is sensitive to $\Gamma$. The relaxation parameter $\gamma_{\mathrm{rlx}}$ plays a different role: it is responsible for achieving a steady state. Furthermore, as explained below it is an essential input in order to predict whether the value of the conductance is $G=G_{\text {spec }}$ or $G=G_{\text {meso }}$.

Algebraic average: Let us assume that we have a large collection of similar rings with possibly a broad thermal population of the energy levels. If the energy landscape is not uniform, it is evident that some rings are likely to absorb energy, while others are not, depending on whether the initial level is strongly coupled to its neighboring levels or not. Accordingly the initial rate of energy absorption (per ring) is obtained by a simple algebraic average. This algebraic average reflects the statistical nature of the preparation and has nothing to do with the nature of the dynamics.

Slow down: When we measure $G$ we are not interested in the transient behavior but rather in the long time behavior. The possible scenarios are illustrated in Fig. 1. One possibility is to have small $\gamma_{\mathrm{rlx}}$. In such case the rate of absorption slows down: in the "long run" the rate of energy absorption is limited by the bottlenecks. [The reader can easily make here an analogy with the dynamics of traffic flow]. We would like to mention that the above scenario is further discussed in the context of a later work [13. There the reader can find an actual numerical simulation that demonstrates the transient from an initial large rate of absorption to a much slower long time rate of absorption.

Mesoscopic circumstances: The above scenario features a crossover from a large absorption rate to a slower absorption rate. The transient is characterized by a time scale that we call $t_{\text {stbl }}$. In a later section we shall determine this time scale and its 
dependence on the EMF: The larger $\dot{\Phi}$ the smaller $t_{\text {stbl }}$. The condition for observing the slow-down is obviously

$$
t_{\text {stbl }}(\dot{\Phi}) \ll \gamma_{\text {rlx }}^{-1}
$$

The rate of absorption for $t \gg t_{\text {stbl }}$ depends on the possibility to make long sequences of transitions. Hence the value of $G$ is smaller compared with the initial anticipation. This value is what we call $G_{\text {meso }}$.

Spectroscopic circumstances: Thus the theory for $G_{\text {meso }}$ becomes relevant whenever the relaxation process is not efficient enough to mask the intrinsic dynamics of the device. Optionally one may say that the EMF should not be too small. What happens if $\dot{\Phi}$ becomes small, such that Eq.(2) breaks down? In such case the relaxation process re-initiated the initial distribution before the slow-down shows up. Consequently the drop in the rate of absorption is avoided. The value of $G$ in the latter circumstances is what we call $G_{\text {spec }}$.

\section{Description of the model system}

For sake of analysis we would like to define the simplest model where our general idea can be demonstrated. As explained in the introduction there is a large class of systems, where the perturbation matrix $\left(\mathcal{I}_{n m}\right)$ is structured. In particular this is the case with ballistic devices. The simplest would be to consider a one channel device driven by EMF, hence $\mathcal{I}_{n m}$ are the matrix elements of the current operator. In order to have some weak scattering mechanism we consider ring which is weakly connected to a big dot region. A particle that moves inside the ring has some small probability to enter into the dot region, where its velocity is randomized. We are going to argue that the long-time energy absorption process is not determined by a simple algebraic average over $\left|\mathcal{I}_{n m}\right|^{2}$, but rather involves a non-trivial coarse graining procedure. We would like to figure out what is the conductance of such ring-dot device as a function of the ring-dot coupling.

To be specific we analyze the model which is illustrated in Fig. 2a, where the dot is modeled as a big chaotic network. The ring states mix with the dense set of the dot states, and resonances are formed. Large $\mathcal{I}_{n m}$ matrix elements are found only between states within resonances, thus leading to a structured band profile (see Fig. 3b). We assume DC driving: this means that the driving frequency is much smaller compared with the energy scales that characterize the structures of $\mathcal{I}_{n m}$. We will show that the off-resonance regions form bottlenecks for the long-time energy absorption. As a result we get $G_{\text {meso }} \ll G_{\text {spec }}$. It should be clear that if $\mathcal{I}_{n m}$ had no structure we would get $G_{\text {meso }}=G_{\text {spec }}$.

In the presented analysis dynamical localization effect is ignored. This mechanism also leads to suppression of energy absorption, but it involves much longer time scales. Moreover, it is extremely sensitive to decoherence, and to any temporal irregularity of the driving. Thus in typical realistic experimental circumstances the suppression of diffusion due to 'bottlenecks' is much more likely than the suppression of diffusion due to a dynamical localization effect.

\section{Main results and their experimental significance}

Before we dive into the derivations, we would like to give an overview of the main results, and to discuss their experimental significance. We also exploit the opportunity 
to comment on the results of a follow-up work [12] where multi-mode chaotic rings have been analyzed.

If the device were opened as in Fig. 2c we could have asked what is its the Landauer conductance. It is convenient to characterize the device by the energy averaged Landauer conductance. The averaging is over the relevant energy range around $E \sim E_{F}$. The energy window of interest is assumed to be classically small but quantum mechanically large (many levels):

$$
g_{c l}=\overline{g_{L}(E)}
$$

We use $0<g<1$ rather than $G$ in order to indicate that the conductance of the is measured in units of $e^{2} /(2 \pi \hbar)$. The subscript of $g_{c l}$ further imply that the energy averaged Landauer conductance yields the classical transmission of the device.

From a theoretical standpoint we can ask what is the "conductance" of the same device if it is integrated in a closed circuit as in Fig. 2b. If we could (hypothetically) ignore the quantum interference within the ring, then we would get (section 9) the "Drude" result

$$
G_{\text {Drude }}=\frac{e^{2}}{2 \pi \hbar}\left[\frac{g_{c l}}{1-g_{c l}}\right]
$$

which diverges in the $g_{c l} \rightarrow 1$ limit. Indeed we are going to argue that a similar result is obtained for the spectroscopic conductance. We say "similar" rather than "identical" because quantum mechanics sets an upper bound to $G$.

If the environmentally induced relaxation is weak, we argue that the spectroscopic result is wrong. Then we have to calculate the mesoscopic conductance using a recipe that we are going to develop in sections 5-8. This leads to

$$
G_{\text {meso }}=\frac{e^{2}}{2 \pi \hbar}\left(1-g_{c l}\right)^{2} g_{c l}
$$

One observes that in the limit $g_{c l} \rightarrow 1$ (weak coupling of the ring with the dot) the mesoscopic conductance goes to zero. This should be contrasted with the behavior of the Drude result.

Measurements of the conductance of closed mesoscopic rings have been performed already 10 years ago 9. In a practical experiment a large array of rings is fabricated. The conductance measurement can be achieved via coupling to a highly sensitive electromagnetic superconducting micro-resonator. In such setup the EMF is realized by creating a current through a "wire" that spirals on top of the array, and the conductance of the rings is determined via their influence on the electrical circuit. Another possibility is to extract the conductance from the rate of Joule heating. The later can be deduced from a temperature difference measurement assuming that the thermal conductance is known.

Ballistic devices are state-of-the-art in mesoscopic experiments. Moreover we believe that molecular size devices with closed "ring geometry" are going to be of great interest in the near future. It is likely that the dynamics is such devices would be of ballistic nature. Namely, it is likely that the mean free path in such rings would be larger compared with their perimeter.

Single-mode rings: The results in the present paper apply to single mode devices with ring-dot geometry. Such a device can be realized in practice. Furthermore, by incorporating a gate, one can control the ring-dot coupling. Hence such geometry 
looks optimal for an experimental test of the theory: The conductance can be measured as a function of the coupling, and at least the qualitative agreement with Eq.(15) can be tested.

Multi-mode rings: In a follow-up work 12 we analyze the mesoscopic conductance of multi-mode chaotic rings. For example one can picture such a device as a wide ring with a small gate-controlled deformation. Thus it is possible to test the theory by measuring $G$ as a function of the gate voltage determined $g_{T}$. It is explained in [12] that also in the case of a multi mode ring the eigenfunctions are nonergodic for $\left(1-g_{T}\right) \ll 1$. Consequently absorption is suppressed due to not having "connected sequences of transitions". It should be noted however that in general there is no simple structure of resonances, in contrast to the 1 mode case that we are going to analyze. Therefore an accurate estimate of the mesoscopic conductance requires a more elaborated resistor network analogy.

\section{The Kubo-Einstein formula for the diffusion}

The purpose of this section is to review a well known expression for the diffusion coefficient $D$. If we were talking about diffusion in real space, then it is well known from any statistical mechanics textbook that $D$ is equal to the integral over the velocityvelocity correlation function. This is known as the Einstein formula. Similarly, the diffusion coefficient in energy space is related to the integral over the current-current correlation function. This is regarded by some authors [11] as a particular version of the Kubo formula, because with some extra assumptions it leads to the "popular" version of the Kubo formula for the conductance $G$.

We define the one-particle current operator $\mathcal{I}$ in the conventional way as the symmetrized version of $e \hat{v} \delta\left(\hat{x}-x_{0}\right)$, with $\hat{v}=\hat{p} / \mathrm{m}$, where $\mathrm{m}$ and $e$ are the mass and the charge of a spinless electron, and $x=x_{0}$ is a section through which the current is measured. In the later numerical analysis it was convenient to re-define $\mathcal{I}:=\int d x_{0} a\left(x_{0}\right) \mathcal{I}$ where $a\left(x_{0}\right)$ is a wide weight function whose integral over the ring obeys $\oint a(x) d x=1$. The current-current correlation function for an electron with energy $E$ is

$$
C_{E}(\tau)=\langle\mathcal{I}(\tau) \mathcal{I}(0)\rangle
$$

It is customary to symmetrize this function, but this is not essential since we later use it within a $d \tau$ integral that goes from $-\infty$ to $\infty$. The power spectrum of the fluctuations is defined as the Fourier transform of the correlation function $C_{E}(\tau)$. In the quantum case it is related to the matrix elements of the current operator as follows [15]:

$$
\tilde{C}_{E}(\omega)=\left[\sum_{n(\neq m)}\left|\mathcal{I}_{n m}\right|^{2} 2 \pi \delta_{\Gamma}\left(\omega-\frac{E_{m}-E_{n}}{\hbar}\right)\right]_{E_{m} \sim E}
$$

where the smoothing parameter $\Gamma$ is introduced because it is required in a later stage.

Having defined the current-current correlation function, we can write the KuboEinstein formula for the EMF-induced diffusion in energy space:

$$
D_{E}=\frac{1}{2} \int_{-\infty}^{\infty} C_{E}(\tau) d \tau \times \dot{\Phi}^{2}
$$


The units of $D_{E}$ are such that $\delta E^{2}=2 D_{E} t$ for a local spreading of a wavepacket. In appendix A we summarize the derivation of this formula and also write the associated diffusion equation that describes the evolution of an arbitrary distribution $\rho(E)$. An optional way to write the Kubo-Einstein formula is

$$
D_{E}=\frac{1}{2} \tilde{C}_{E}(\omega=0) \times \dot{\Phi}^{2}
$$

We immediately see that in the quantum mechanical case the Kubo-Einstein formula is ill-defined unless we specify the parameter $\Gamma$. Note that for $\Gamma=0$ we get formally $D_{E}=0$. The physics behind this formula becomes more transparent if we adopt the Fermi-Golden rule picture as discussed in the next section.

\section{The Fermi Golden Rule Picture}

The Hamiltonian of the ring system in the adiabatic basis is

$$
\mathcal{H} \mapsto E_{n} \delta_{n m}+W_{n m}
$$

where

$$
W_{n m}=i \dot{\Phi} \frac{\hbar \mathcal{I}_{n m}}{E_{n}-E_{m}}
$$

is the perturbation matrix. If the EMF is non-zero then there are transitions between levels. Both Linear Response Theory, and also our extended theory of mesoscopic conductance assume that the rate of transition from an initial level $(m)$ to some other level $(n)$ is determined by Fermi Golden Rule (FGR):

$$
w_{n m}=\frac{2 \pi}{\hbar} \delta_{\Gamma}\left(E_{n}-E_{m}\right)\left|W_{n m}\right|^{2}
$$

Note that upon summation over all transitions to all the levels $n$, the delta function is replaced by the smoothed density of states $\varrho(E)$.

In the adiabatic regime, the level broadening $\Gamma$ is smaller compared with the mean level spacing $\Delta$. In such case the FGR mechanism can be neglected $\left(w_{n m} \sim 0\right)$, and the leading dissipation mechanism, depending on the effectiveness of the environmental relaxation process, is either the Landau-Zener mechanism [11, or the Debye relaxation mechanism [2]. In the present work we assume that $\Gamma$ is much larger than $\Delta$, but much smaller compared with any other semiclassical energy scale. This implies that FGR transitions are the dominant mechanism for diffusion in energy space.

Due to the FGR transitions there is a diffusion in energy space. The local diffusion rate is:

$$
D_{E}=\frac{1}{2} \sum_{m}\left(E_{n}-E_{m}\right)^{2} w_{n m}=\pi \hbar \varrho(E) \overline{\left|\mathcal{I}_{n m}\right|^{2}} \times \dot{\Phi}^{2}
$$

The first expression in Eq. (13) is just as in the standard analysis of a random walk problems. Upon substitution of the FGR expression it leads to Eq.(9). The second expression in Eq. (13) is just a loose way to re-write Eq. (9). The dependence on $\Gamma$ is implicit. 


\section{Coarse grained diffusion}

The probability distribution in energy space obeys a diffusion equation (see appendix A). If we change form the variable $E$ to the variable $n$ the diffusion equation takes a simpler form:

$$
\frac{\partial \rho(n)}{\partial t}=\frac{\partial}{\partial n}\left(D_{n} \frac{\partial}{\partial n} \rho(n)\right)
$$

where $D_{n}=\varrho^{2} D_{E}$. Our main motivation to re-write the diffusion equation in the $n$ variable is to suggest the analogy with the familiar problem of random walk on a lattice where $n$ is re-interpreted as a site index. In the standard textbook discussion $D_{n}$ is uniform all over space. But what happens if $D_{n}$ has some microscopic modulation? We would like to argue that in such case the coarse grained diffusion coefficient is given by an harmonic average over the local $D_{n}$. Namely,

$$
D=\left\langle\left\langle D_{n}\right\rangle\right\rangle \equiv\left[\lim _{N \rightarrow \infty} \frac{1}{N} \sum_{n}^{N} D_{n}^{-1}\right]^{-1}
$$

This is like calculating the resistivity of a random network of resistors in one dimension. The summation can be re-interpreted as the addition of resistors in series. This analogy is further developed in a subsequent work [13. Below we derive this result using the diffusion picture language. It is important to realize that the validity of the harmonic average recipe Eq. (15) is limited. It is assumed that $D_{n}$ has a smooth modulation as a function of with $n$. Otherwise $n$ cannot be treated as a continuous variable, and one should use the more elaborated "random network" scheme of calculation.

In order to keep consistency of notations we turn back to use $E$ as the diffusion space variable, as in appendix A. For simplicity we assume that locally the smoothed density of states is constant. Hence the diffusion equation is Eq. (14) with $n$ replaced by $E$. It can be regarded as a continuity equation

$$
\frac{\partial \rho(E)}{\partial t}=-\frac{\partial}{\partial E} J_{E}
$$

where the probability current (probability transported per unit time) is given by Fick's law

$$
J_{E}=-D_{E} \frac{d \rho(E)}{d E}
$$

In order to determine the coarse grained diffusion we assume a steady state distribution $\rho(E)$ that supports a current $J_{E}=$ const. It follows that

$$
\rho\left(E_{2}\right)-\rho\left(E_{1}\right)=-\int_{E_{1}}^{E_{2}}\left(J_{E} / D_{E}\right) d E
$$

This implies that the coarse-grained diffusion coefficient is given by

$$
D^{-1}=-\frac{1}{J_{E}} \frac{\rho\left(E_{2}\right)-\rho\left(E_{1}\right)}{E_{2}-E_{1}}=\frac{1}{E_{2}-E_{1}} \int_{E_{1}}^{E_{2}} D_{E}^{-1} d E
$$

where $E_{2}-E_{1}$ is the coarse graining scale. Hence we get the desired result: the coarse grained diffusion coefficient $D$ is obtained by harmonic average over the modulated $D_{E}$. A concise way to write this result for the coarse grained diffusion is

$$
D=\left\langle\left\langle D_{E}\right\rangle\right\rangle=\left[\overline{1 / D_{E}}\right]^{-1}
$$


where the indicated average in the r.h.s. is over the energy $E$. This observation is going to be the corner stone in the analysis of the mesoscopic conductance. Needless to say that the harmonic average recipe implies vanishing $D$ if $D_{E}$ has bottlenecks.

\section{The diffusion-dissipation relation}

There is a simple relation between the diffusion and the rate of energy absorption. For a derivation of this relation see appendix A. In the case of a low temperature Fermi occupation it takes the form

$$
\dot{\mathcal{W}}=\varrho\left(E_{F}\right)\left\langle\left\langle D_{E}\right\rangle\right\rangle
$$

where $E_{F}$ is the Fermi energy. By substitution of Eq.(9) and comparing with Eq.(11) we deduce the following expression for the conductance:

$$
G=\varrho\left(E_{F}\right) \times \frac{1}{2}\left\langle\left\langle\tilde{C}_{E}(\omega=0)\right\rangle\right\rangle
$$

From the discussion in section 2 it is implied that in the case of the spectroscopic conductance $G_{\text {spec }}$, the energy averaging $\langle\langle.\rangle$.$\rangle should be algebraic. In the case of$ diffusive rings it is customary to replace the (algebraic) energy averaging by disorder averaging. In contrast to that from the discussion in section 7 it is implied that in the case of the mesoscopic conductance $G_{\text {meso }}$, the energy averaging $\langle\langle.\rangle$.$\rangle should$ be harmonic. To be more precise, the harmonic average applies if $D_{E}$ has smooth modulation as a function of $E$. This is going to be the case with our simple example. In more complicated circumstances (e.g. multimode chaotic rings) the coarse graining should be done using a somewhat more elaborated resistor network analogy [13, 12].

In the case of $G_{\text {spec }}$ calculation, Eq.(21) is just the conventional Kubo formula. If the energy landscape is uniform then the distinction between $G_{\text {spec }}$ and $G_{\text {meso }}$ is not important. But if $\mathcal{I}_{n m}$ is sparse or structured then $G_{\text {meso }}$ might be much smaller compared with $G_{\text {spec }}$.

Sensitivity to level broadening: The parameter $\Gamma$ is an implicit input for the calculation. As discussed in section 6 we assume that there is effectively a quasicontinuum $(\Gamma \gg \Delta)$. The spectroscopic conductance $G_{\text {spec }}$ is not very sensitive to $\Gamma$. For example, in the case of diffusive rings the weak localization corrections are of order $(\Gamma / \Delta)^{-1}$. In contrast to that $G_{\text {meso }}$ is extremely sensitive to $\Gamma$. By increasing $\Gamma$ we can enhance the diffusion by several orders of magnitudes. As common in the traditional LRT treatment $[8]$ also here $\Gamma$ is going to be a free parameter in the theory.

\section{Semiclassical considerations}

If the current-current correlation function is calculated classically, one observes a and very slow monotonic dependence on $E$. Since the practical interest is in a classically

small energy window, this dependence can be ignored. For a one dimensional ring whose classical transmission is $g_{c l}$ the correlation function $C_{E}(\tau)$ decays exponentially. The intensity of the fluctuations $\tilde{C}_{E}(\omega=0)$ that appears in Eq.(21) equals the area under this classical correlation function leading to [10]:

$$
G_{\text {Drude }}=\frac{e^{2}}{2 \pi \hbar}\left(\frac{g_{c l}}{1-g_{c l}}\right)
$$

This is simply the Drude result being written in terms of $g_{c l}$ instead in terms of the mean free path $\ell \approx L_{0} /\left(1-g_{c l}\right)$, where $L_{0}$ is the length of the ring. We note that if 
we had neglected the multiple reflections within the ring (which is formally justified in case of an open system) we would get $g_{c l}$ instead of $g_{c l} /\left(1-g_{c l}\right)$ in agreement with the Landauer formula.

The "classical" result is obtained also within the framework of a semiclassical Green function calculation [16] that employs a diagonal approximation. Such calculation assumes an algebraic average over the energy or if applicable, over realizations of disorder. We recall that algebraic average is justified for the purpose of calculating the spectroscopic conductance. Still the spectroscopic conductance cannot diverge in the limit $g_{c l} \rightarrow 1$. We shall discuss the upper bound on $G_{\text {spec }}$ in section 12 .

As stated above in the classical analysis $C_{E}(\tau)$ and hence $D_{E}$ are essentially independent of $E$. But upon quantization $D_{E}$ might have a modulation on a classically small energy scale $\Delta_{0} \propto \hbar$ which is still much larger compared with the mean level spacing $\Delta$. The energy scale $\Delta_{0}$ reflects the appearance of a structured band profile and it is the new ingredient in our analysis. The time to explore this energy scale is

$$
t_{\mathrm{stbl}}=\frac{\Delta_{0}^{2}}{\left\langle\left\langle D_{E}\right\rangle\right\rangle} \propto \dot{\Phi}^{-2}
$$

Assuming that $D_{E}$ has indeed a "microscopic" dependence on $E$, and assuming that Eq.(2) is satisfied, we have argued that the long time energy absorption is determined by the mesoscopic conductance, which involves an harmonic average. Consequently the mesoscopic conductance is typically smaller than the spectroscopic conductance, and does not correspond to the classical (Drude) result!

\section{Preliminary analysis of the model system}

We turn now to a detailed description of our model (Fig. 2a). The dot region is modeled as a chaotic network which is weakly coupled to a ring. The network is attached with two "legs" in order to destroy odd-even symmetries of the eigenstates. Each coupling vertex is described by a $3 \times 3$ symmetric orthogonal matrix (the "splitter" of Ref. [17]):

$$
\boldsymbol{S}=\left(\begin{array}{ccc}
\frac{1}{2}\left(1-\sqrt{1-2 c^{2}}\right) & \frac{1}{2}\left(1+\sqrt{1-2 c^{2}}\right) & c \\
\frac{1}{2}\left(1+\sqrt{1-2 c^{2}}\right) & \frac{1}{2}\left(1-\sqrt{1-2 c^{2}}\right) & -c \\
c & -c & \sqrt{1-2 c^{2}}
\end{array}\right)
$$

where the coupling parameter is $0<c<1$. Once the chaotic network is integrated into the ring one can regard it as a "black box" which is characterized by a $2 \times 2$ scattering matrix. This $2 \times 2$ scattering matrix is characterized by the average Landauer conductance $g_{c l}$. Hence the coupling between the network and the ring is quantified by the dimensionless parameter $1-g_{c l} \approx c^{2}$ (see Fig. 4b), which we assume to be much smaller than 1 .

The length of the ring is $L_{0}$ while the total length of all the bond is $L$. Resonances are formed because of the coupling of the ring states to the network states. Hence we have

$$
\begin{aligned}
\Delta & =\hbar v_{E} \frac{\pi}{L}=\text { mean level spacing } \\
\Delta_{0} & =\hbar v_{E} \frac{\pi}{L_{0}}=\text { distance between resonances } \\
\Gamma_{0} & =\frac{1}{2 \pi}\left(1-g_{c l}\right) \Delta_{0}=\text { width of resonances }
\end{aligned}
$$


where $v_{E}=(2 E / \mathrm{m})^{1 / 2}$. Having defined the dimensionless parameters of the model system $\left(L / L_{0}\right.$ and $\left.g_{c l}\right)$ as well as the relevant energy scales $\left(\Delta, \Delta_{0}, \Gamma_{0}\right)$, we are fully equipped to turn to the calculation of the conductance.

A key object in the analysis is the weight $q\left(E_{n}\right)$ of each state in the ring region. We write the wavefunction on the ring as $\psi^{n}(x \in \operatorname{ring})=\mathrm{A}_{n} \sin \left(\varphi_{n}+k_{n} x\right)$ and define

$$
q\left(E_{n}\right)=\frac{L}{2}\left|\mathrm{~A}_{n}\right|^{2} \approx \frac{L}{L_{0}} \sum_{r}|\langle r \mid n\rangle|^{2}
$$

where $r$ are the ring states in the absence of coupling $(c=0)$. If we could assume ergodicity of the wavefunctions then $A_{n}=(2 / L)^{1 / 2}$ and we would get $q(E)=1$. But if the coupling becomes weak $\left(1-g_{c l} \ll 1\right)$ then ergodicity does not hold (Fig. 4a) and we have $\bar{q}=1$ only upon averaging over energy. Using perturbation theory we get as the simplest approximation that $q(E)$ is a sum of Lorentzian:

$$
q(E) \approx \frac{\Delta_{0}}{\pi} \sum_{r} \frac{\left(\Gamma_{0} / 2\right)}{\left(\Gamma_{0} / 2\right)^{2}+\left(E-\varepsilon_{r}\right)^{2}}
$$

where the resonance energies $\varepsilon_{r}$ have spacing $\Delta_{0}$. It is important to notice that the above expression does not reflect that

$$
\operatorname{maximum}\left[q\left(E_{n}\right)\right]=\frac{L}{L_{0}}=\frac{\Delta_{0}}{\Delta}
$$

The maximum corresponds to the extreme case of having all the probability of the wavefunction inside the ring such that $A_{n}=\left(2 / L_{0}\right)^{1 / 2}$. This situation is attained if we decrease the coupling so as to have no mixing of ring states with dot states $\left(\Gamma_{0} \sim \Delta\right)$. In the following discussion we assume that the latter (trivial possibility) is not the case. Hence we observe from Eq. (28) that $q(E) \approx\left(1-g_{c l}\right)^{-1}$ on resonances (where $E \sim \varepsilon_{r}$ ), while $q(E) \approx\left(1-g_{c l}\right.$ ) off resonances (where $\left|E-\varepsilon_{r}\right| \sim \Delta_{0} \gg \Gamma_{0}$ ). The algebraic energy average over $q^{2}(E)$ is dominated by the peaks within resonance regions, whereas the harmonic average is dominated by the bottleneck off-resonance (valley) regions. The relative size of the resonance regions is $\Gamma_{0} / \Delta_{0} \approx\left(1-g_{c l}\right)$. Therefore we get

$$
\begin{aligned}
\overline{q^{2}} & \approx\left(1-g_{c l}\right)^{-1} \\
{\left[\overline{1 / q^{2}}\right]^{-1} } & \approx\left(1-g_{c l}\right)^{2}
\end{aligned}
$$

\section{Calculation of the conductance for the model system}

Given the eigenfunctions of the network the matrix elements of $\mathcal{I}$ are

$$
\mathcal{I}_{n m} \approx-i \frac{e v_{\mathrm{F}}}{2} \mathrm{~A}_{n} \mathrm{~A}_{m} \sin \left(\left[\varphi_{n}-\varphi_{m}\right]+\left[k_{n}-k_{m}\right] x_{0}\right) .
$$

Without loss of generality we set $x_{0}=0$ and find

$$
\frac{2 \pi \hbar}{\Delta}\left|I_{n m}\right|^{2}=e^{2} \frac{v_{\mathrm{F}}}{L} q\left(E_{n}\right) q\left(E_{m}\right) \times g_{\varphi} \quad \text { for }\left|E_{n}-E_{m}\right| \ll \Delta_{0}
$$

where $0<g_{\varphi}<1$ is defined as the average value of $2\left|\sin \left(\varphi_{n}-\varphi_{m}\right)\right|^{2}$ for nearby levels If we change $x_{0}$ the correction to $g_{\varphi}$ is at most of order $\left(\left(E_{n}-E_{m}\right) / \Delta_{0}\right)^{2}$ and hence can be neglected. Now we can get an explicit result for $\tilde{C}_{E}(\omega)$ via Eq. (17) and hence for the conductance:

$$
G=\frac{e^{2}}{2 \pi \hbar}\left\langle\left\langle q(E) q_{\Gamma}(E+\hbar \omega)\right\rangle\right\rangle \times g_{\varphi}
$$


where $q_{\Gamma}(E)$ is a smoothed version of $q(E)$ as implied by Eq. (17). Note that in the numerical analysis one should be careful to use a smoothing kernel that excludes the center element, as implied by the restriction $m \neq n$.

Using Eq. (33) with Eq. (30) we get for the spectroscopic conductance

$$
G_{\mathrm{spec}}=\left[\frac{e^{2}}{2 \pi \hbar}\right]\left(1-g_{c l}\right)^{-1} g_{\varphi}
$$

Comparing this to Eq. (22) we deduce $g_{\varphi}=g_{c l}$. On the other hand using Eq.(33) with Eq.(31) we get

$$
G_{\text {meso }}=\left[\frac{e^{2}}{2 \pi \hbar}\right]\left(1-g_{c l}\right)^{2} g_{\varphi}
$$

which leads to Eq.(5) for the mesoscopic conductance. We can re-phrase the above procedure as follows: Given the well behaved ( $\Gamma$ insensitive) spectroscopic conductance, the mesoscopic conductance is

$$
G_{\text {meso }}=\left[\left(\overline{1 / q^{2}}\right)^{-1} / \overline{q^{2}}\right] \times G_{\text {spec }}
$$

with $\Gamma$ implicit in the definition of $q$. Thus in order to calculate $G_{\text {meso }}$ we need to know both $G_{\text {spec }}$ and $q_{\Gamma}(E)$. For flat band $(q(E)=1)$ and we get $G_{\text {meso }}=G_{\text {spec }}$, but in general $G_{\text {meso }}<G_{\text {spec }}$.

\section{Numerics}

Numerical tests have been done for the model system of Fig. 2a. We have chosen a network containing 25 bonds of length $\sim 1$ such that $L \approx 25$. Our energy window was set around $k \sim 25000$. We computed $\left(k_{n}, \varphi_{n}, \mathrm{~A}_{n}\right)$ and with these data we have done the analysis described above. In particular we have obtained $q(E)$ for various values of the couplings (see Fig. 4a). Then we have calculated both the algebraic and the harmonic averages of $q^{2}(E)$. From the results which are presented in Fig. $4 \mathrm{~b}$ we were able to deduce that the relation between these averages and $g_{c l}$ is as expected from the theoretical considerations.

In Fig. 5 we show that the numerical result for $G_{\text {spec }}$ and $G_{\text {meso }}$ is sensitive to the smoothing parameter $\Gamma$. Note that the displayed range $\Gamma$ exceeds the physically relevant region. In the adiabatic regime $(\Gamma \leq \Delta)$ the results are unstable numerically and of no significance. Also very large values of $\Gamma$ are not physically significant.

We observe in Fig. 5 that $G_{\text {spec }}$ cannot exceed the quantum bound, and becomes saturated in the limit $g_{c l} \rightarrow 1$. This saturation is attained for $\left(1-g_{c l}\right)^{-1}>L / L_{0}$ as implied by the quantum border of Eq.(29):

$$
\left.G_{\text {spec }}\right|_{\text {maximum }}=\left[\frac{e^{2}}{2 \pi \hbar}\right] \frac{L}{L_{0}}
$$

We have a second set of data (not displayed) for a similar network with $L / L_{0}$ larger by a factor 3.5. We have verified that the saturation value of $G_{\text {spec }}$ increases by the same factor. 


\section{Summary}

The original motivation for this work was to study the simplest model of a closed device where the FGR picture of transitions and the notion of conductance are meaningful. In order to construct such a model quantum chaos considerations are essential. A ring with a simple scatterer is not good enough: Energy diffusion cannot be justified due to adiabaticity or strong localization effect [5]. Therefore we have replaced the scatterer by a complicated network, which has allowed us to define circumstance such that the FGR picture would apply. Our initial inclination was to assume that in such circumstance we could use the Kubo formula in order to calculate the rate of energy absorption. But then we have realized that the applicability of the FGR picture is not enough in order to get Kubo. This turned out to be an example for a system where the conductance depends crucially on the non-universal structure of the perturbation matrix.

In other systems with structured band profile a similar effect is expected, although not necessarily as dramatic as in our simple model. In particular we note a subsequent work [12] where we calculate the conductance of multi mode ballistic rings. Thus $a$ feature that looked like an anomaly of a single mode device, has turned out to be a general theme in the theory of energy absorption. A further non-trivial extension of the theory has allowed also to calculate the absorption of small metallic grains that are irradiated by a low frequency noise source [13.

The model system that we have picked is of particular interest because it adds a twist to the old discussion of the Landauer formula 20. For an open system the two terminal conductance of a one-channel system is less than unity. For a closed (zero terminal) device we have to distinguish between spectroscopic and mesoscopic conductance. The former equals $g_{c l} /\left(1-g_{c l}\right)$ and can be very large in the limit of weak scattering, while the latter equals $\left(1-g_{c l}\right)^{2} g_{c l}$ and goes to zero in this limit (disregarding higher-order corrections). More generally we can say that within the validity limits of our assumptions, $g_{c l}$ is the upper limit for the mesoscopic conductance of a single-mode system. There is a numerical indication [12, not yet conclusive, that also in the case of a multimode ring the mesoscopic conductance is limited by the number of open modes.

The calculation of the conductance of closed devices is sensitive to the level broadening parameter $\Gamma$. Hence theoretical considerations that go well beyond the common Kubo formalism are essential. $\Gamma$ is determined by the non-adiabaticity of the driving [14 and/or by the surrounding environment 3]. We have assumed in the present paper that $\Delta \ll \Gamma \ll \Delta_{0}$. Our results do not apply to the adiabatic regime where other (rival) dissipation mechanisms apply (Landau-Zener [5], Debye [2]). On the other extreme, for very large driving rate (EMF) we may have a nonperturbative response [18, that can invalidate the fluctuation-diffusion relation on which the calculation of $D_{E}$ has been based. 


\section{Appendix A. The Kubo-Einstein and the diffusion-dissipation relations}

In this appendix we review the derivation of the Kubo-Einstein formula and the diffusion-dissipation relation following [11, 19]. The diffusion in energy space is deduced from the relation

$$
\frac{d \mathcal{H}}{d t}=\frac{\partial \mathcal{H}}{\partial t}=\dot{\Phi} \times \frac{\partial \mathcal{H}}{\partial \Phi}=-\dot{\Phi} \times \mathcal{I}
$$

This exact relation holds both classically and quantum mechanically. In the latter case we have to use Heisenberg picture. For simplicity of presentation we use a classical language and write

$$
E(t)-E(0)=-\dot{\Phi} \int_{0}^{t} \mathcal{I}\left(t^{\prime}\right) d t^{\prime}
$$

averaging over an initial microcanonical preparation we get

$$
\left\langle(E(t)-E(0))^{2}\right\rangle=\dot{\Phi}^{2} \int_{0}^{t} \int_{0}^{t}\left\langle\mathcal{I}\left(t^{\prime}\right) \mathcal{I}\left(t^{\prime \prime}\right)\right\rangle_{E} d t^{\prime} d t^{\prime \prime}
$$

Thus

$$
\delta E^{2}(t)=2 D_{E} t
$$

where $D_{E}$ is given by Eq. (8). On long times one can argue [21] that the probability distribution $\rho(E)$ of the energy should satisfies the following diffusion equation:

$$
\frac{\partial \rho}{\partial t}=\frac{\partial}{\partial E}\left(\varrho(E) D_{E} \frac{\partial}{\partial E}\left(\frac{1}{\varrho(E)} \rho\right)\right)
$$

where $\varrho(E)$ is the density of states. The energy of the system is $\langle\mathcal{H}\rangle=\int E \rho(E) d E$. It follows that the rate of energy absorption is

$$
\dot{\mathcal{W}}=\frac{d}{d t}\langle\mathcal{H}\rangle=-\int_{0}^{\infty} d E \varrho(E) D_{E} \frac{\partial}{\partial E}\left(\frac{\rho(E)}{\varrho(E)}\right)
$$

For a Fermi occupation $\rho(E)=\varrho(E) f(E)$ where $f(E)$ is the occupation function. At zero temperature we get Eq.(20). 


\section{Acknowledgments}

DC thanks Michael Wilkinson, Bernhard Mehlig, Yuval Gefen and Shmuel Fishman for intriguing discussions. The preparation of the expanded version of this paper has been inspired by the helpful suggestions of Miriam Blaauboer, and we thank Boris Shapiro and Markus Büttiker for their additional comments. The research was supported by the Israel Science Foundation (grant No.11/02), and by a grant from the GIF, the German-Israeli Foundation for Scientific Research and Development.

\section{References}

[1] M. Büttiker, Y. Imry and R. Landauer, Phys. Lett. 96A, 365 (1983).

[2] The Debye relaxation mechanism is discussed by

R. Landauer and M. Büttiker, Phys. Rev. Lett. 54, 2049 (1985).

M. Büttiker, Phys. Rev. B 32, 1846 (1985).

M. Büttiker, Annals of the New York Academy of Sciences, 480, 194 (1986).

[3] Y. Imry and N.S. Shiren, Phys. Rev. B 33, 7992 (1986).

[4] N. Trivedi and D. A. Browne, Phys. Rev. B 38, 9581 (1988).

[5] Y. Gefen and D. J. Thouless, Phys. Rev. Lett. 59, 1752 (1987). M. Wilkinson, J. Phys. A 21 (1988) 4021.

M. Wilkinson and E.J. Austin, J. Phys. A 23, L957 (1990).

[6] B. Reulet and H. Bouchiat, Phys. Rev. B 50, 2259 (1994).

[7] A. Kamenev, B. Reulet, H. Bouchiat, and Y. Gefen, Europhys. Lett. 28, 391 (1994).

[8] For a review see "(Almost) everything you always wanted to know about the conductance of mesoscopic systems" by A. Kamenev and Y. Gefen, Int. J. Mod. Phys. B9, 751 (1995).

[9] Measurements of conductance of closed diffusive rings are described by B. Reulet M. Ramin, H. Bouchiat and D. Mailly, Phys. Rev. Lett. 75, 124 (1995).

[10] D. Cohen and Y. Etzioni, J. Phys. A 38, 9699 (2005).

[11] M. Wilkinson, J. Phys. A 21, 4021, (1988).

[12] S. Bandopadhyay, Y. Etzioni and D. Cohen, "The conductance of a multi-mode ballistic ring: beyond Landauer and Kubo", cond-mat/0603484

[13] M. Wilkinson, B. Mehlig and D. Cohen, Europhysics Letters 75, 709 (2006).

[14] The $\Gamma$ issue is best discussed in Section VIII of D. Cohen, Phys. Rev. B 68, 155303 (2003).

[15] M. Feingold and A. Peres, Phys. Rev. A 34, 591 (1986). Feingold, D. Leitner, and M. Wilkinson, Phys. Rev. Lett. 986 (1991).

[16] D. Cohen, T. Kottos and H. Schanz, Phys. Rev. E 71, 035202(R) (2005).

[17] M. Büttiker, Y. Imry and M. Ya. Azbel, Phys. Rev. A 30, 1982 (1984).

[18] D. Cohen, Phys. Rev. Lett. 82, 4951 (1999). D. Cohen and T. Kottos, Phys. Rev. Lett. 85, 4839 (2000).

[19] D. Cohen, Annals of Physics 283, 175 (2000).

[20] Y. Imry, Introduction to Mesoscopic Physics (Oxford Univ. Press 1997), and references therein. Optionally see D. Stone and A. Szafer, http://www.research.ibm.com/journal/rd/323/ibmrd3203I.pdf

[21] C. Jarzynski, Phys. Rev. Lett. 74, 2937 (1995); Phys. Rev. E 48, 4340 (1993).

[22] D. Cohen and T. Kottos, Phys. Rev. E 63, 36203 (2001). 

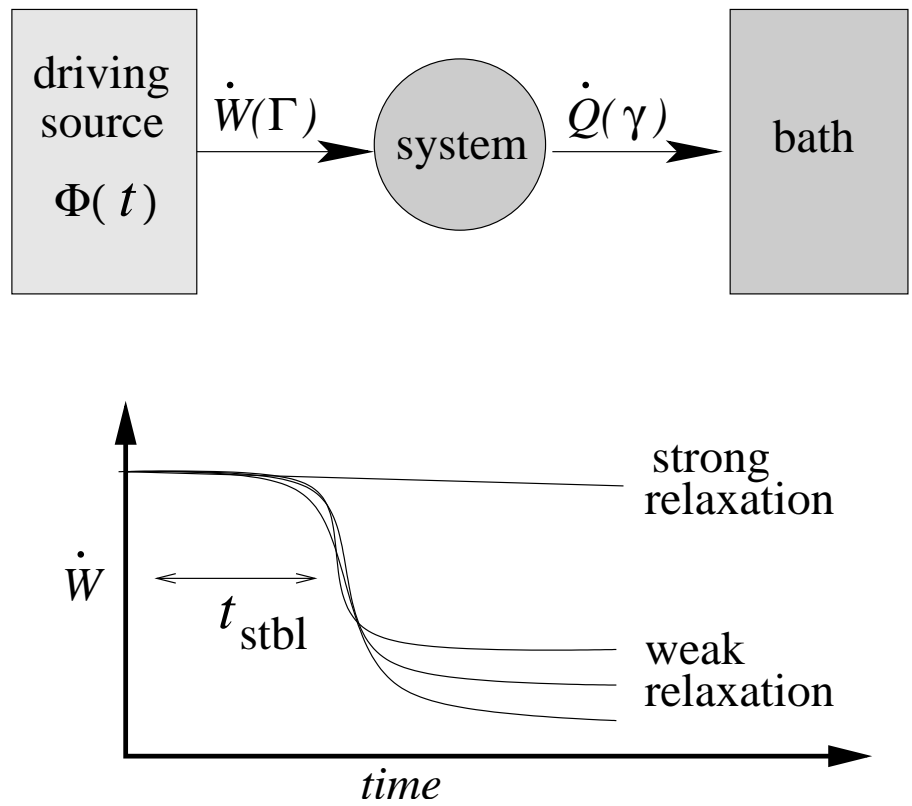

Fig.1: The phenomenology of energy absorption. In the upper panel we present a block diagram. We highlight the rate of energy absorption $\dot{\mathcal{W}}$ which depends on the energy broadening parameter $\Gamma$. We also highlight the rate $\dot{\mathcal{Q}}$ of "heat flow" to the bath, which depends on the energy relaxation rate $\gamma_{\mathrm{rlx}}$. In the lower panel we plot how $\dot{W}$ depends on time. If $\gamma_{\mathrm{rlx}}$ is small than there is a transient to a slower absorption rate that depends on $\Gamma$.
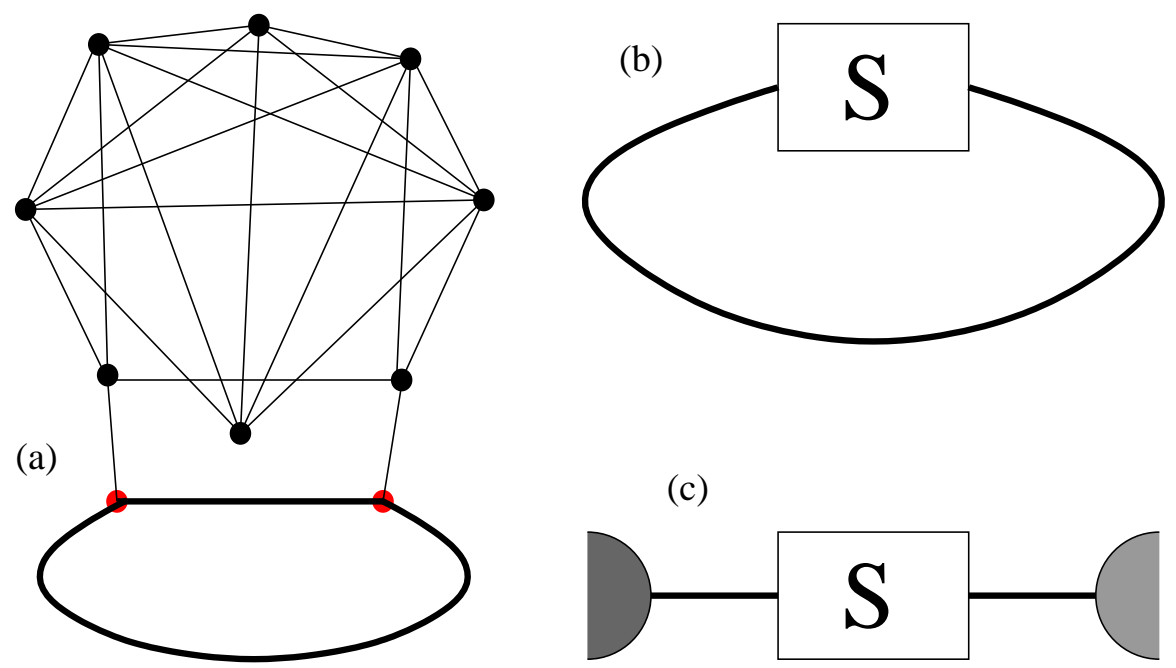

Fig.2: (a) The ring-dot network model. (b) Schematic representation. (c) The corresponding open system. 

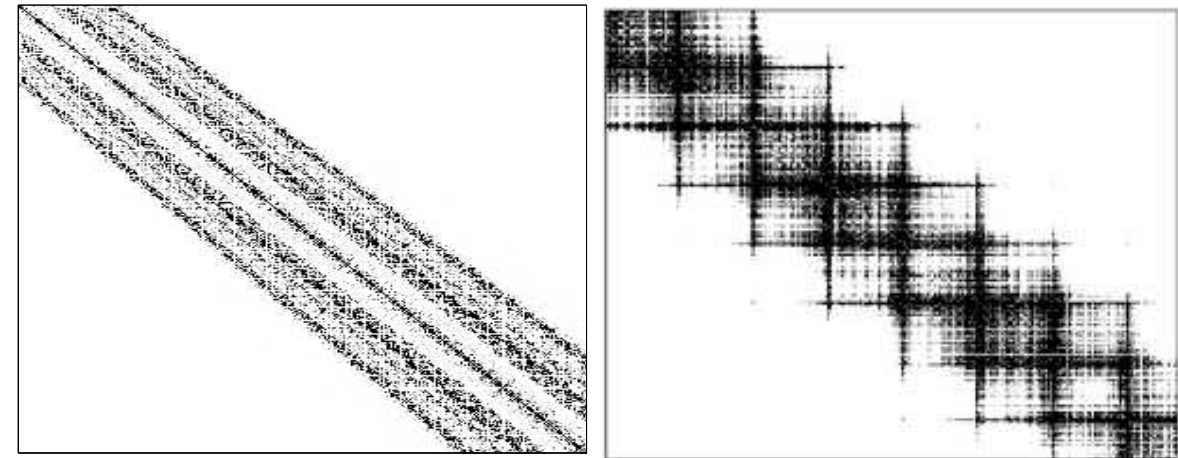

Fig.3: Writing the perturbation in the basis of the unperturbed Hamiltonian we get a structured matrix: On the left we display a representative result for a quantized hard chaos system (taken form Ref. 22]). On the right we display the result for the ring-dot network model of this paper.
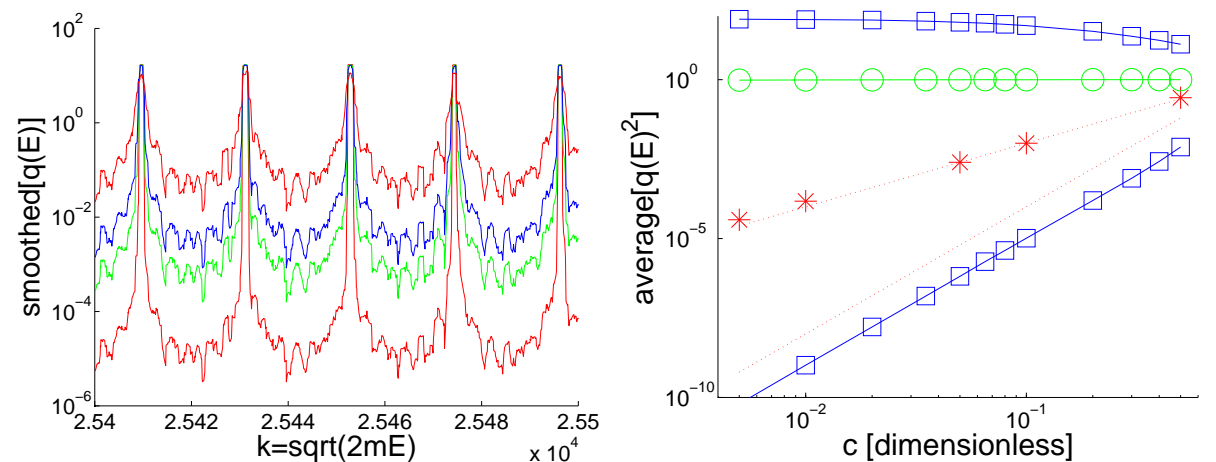

Fig.4: Left panel: Smoothed $q\left(E_{n}\right)$ for $0.005<c<0.5$. Right panel: The horizontal axis is $c$. The dotted red lines are $c^{2}$ and $c^{4}$. The averages $1 / \overline{\left[1 / q(E)^{2}\right]}$ (lower curve), and $\overline{\left[q(E)^{2}\right]}$ (upper curve), and $\overline{[q(E)]}$ (circles) are calculated. The coupling measure $1-g_{c l}$ (stars) is numerically obtained from the Landauer conductance.
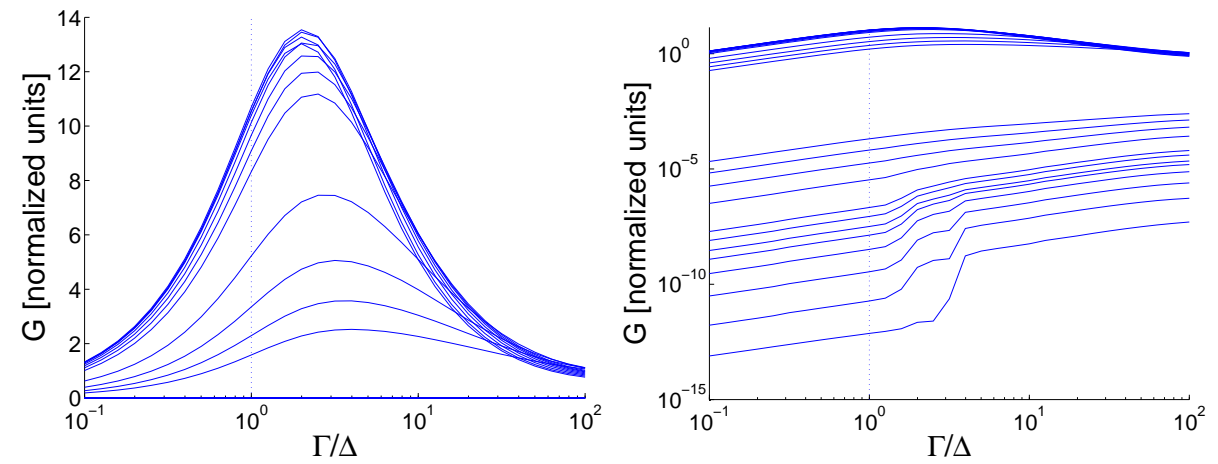

Fig.5: The spectroscopic and mesoscopic conductances as a function of $\Gamma / \Delta$ for the various values of $c$. The former is resolved in the normal scale (left) while the latter is resolved in the log scale (right). The vertical dotted line indicates the $\Gamma$ value that has been assumed in Fig. $4 \mathrm{~b}$. 